Portland State University

PDXScholar

\title{
Effect of Mitochondrial Dysfunction on Age-Related Subcellular Damage \& Autofluoresence in C. elegans
}

Ashleigh R. Mustain

Portland State University

Follow this and additional works at: https://pdxscholar.library.pdx.edu/honorstheses

Let us know how access to this document benefits you.

\section{Recommended Citation}

Mustain, Ashleigh R., "Effect of Mitochondrial Dysfunction on Age-Related Subcellular Damage \& Autofluoresence in C. elegans" (2017). University Honors Theses. Paper 408.

https://doi.org/10.15760/honors.404

This Thesis is brought to you for free and open access. It has been accepted for inclusion in University Honors Theses by an authorized administrator of PDXScholar. Please contact us if we can make this document more accessible: pdxscholar@pdx.edu. 


\title{
Effect of Mitochondrial Dysfunction on Age-Related Subcellular Damage \& Autofluoresence in C. elegans
}

\author{
Ashleigh Mustain, Alexandra Basler, Suzanne Estes, Portland State University, Department of
} Biology

\begin{abstract}
:
Despite considerable research, the patterns and causes of senescence (biological aging) and organismal death are still mysterious. Recent experimental evidence suggests that organismal death in the model nematode, Caenorhabitis elegans, is accompanied by a burst of blue "death fluorescence" (DF), which is generated within intestinal cells by the necrotic cell death pathway [1]. Simultaneously, uranin fluorescence, which can serve as a marker for loss of membrane integrity in lysosome related organelles due to its quenching at low $\mathrm{pH}$, also increases. Dequenching of uranin fluorescence upon gut granule permeabilization has been proposed to lead to a burst of green fluorescence in C. elegans [1]. The same study [1] showed that red autofluorescence exhibits a near linear increase in aging nematodes, thus providing a good biomarker for chronological age. This thesis project investigated whether mitochondrial dysfunction, as represented by the gas-1 (fc21) gene mutation, leads to a different pattern of agerelated uranin (green) fluorescence and red autofluorescence when compared to wildtype. To measure uranin fluorescence, confocal microscopy was used to image age-synchronous samples of gas-1 and N2 wildtype worms dyed with fluorescein sodium salt at three different time points in their life cycle. The same methods were applied to a set of experimentally-evolved lines that were initiated from the gas-1 mutant and adapted to the laboratory for 60 generations. Results indicated differences in age-related patterns of uranin fluorescence between the gas-1 and N2 control strains and divergence of the evolved lines from their mutant ancestor. Both control strains
\end{abstract}


showed the expected pattern of age-related increase in red autofluorescence, which was not shared by the evolved lines. Indeed, no consistent age-associated patterns emerged in either uranin or red fluorescence for evolved lines and no relationship between either type of fluorescence and fitness was observed.

\section{Introduction:}

While much research has been conducted toward uncovering the mechanisms of cell death and apoptosis [2], there is still little known regarding the mechanisms underlying organismal death [1]. It has been proposed that organismal death results from a cascade of cell death [2]. While necrotic cell death has typically been viewed as uncontrolled as opposed to apoptotic (controlled) cell death, evidence has been presented that necrotic cell death can be a regulated process as well [1-6]. The necrotic cell death pathway is characterized by an influx of calcium into the cell post cellular insult which leads to protease (calpain) activation, lysosomal membrane permeabilization (LMP), lysosomal lysis and cytosolic acidosis, and activation of lysosomal proteases (cathepsins) [1; 7-8; Fig. 1]. 


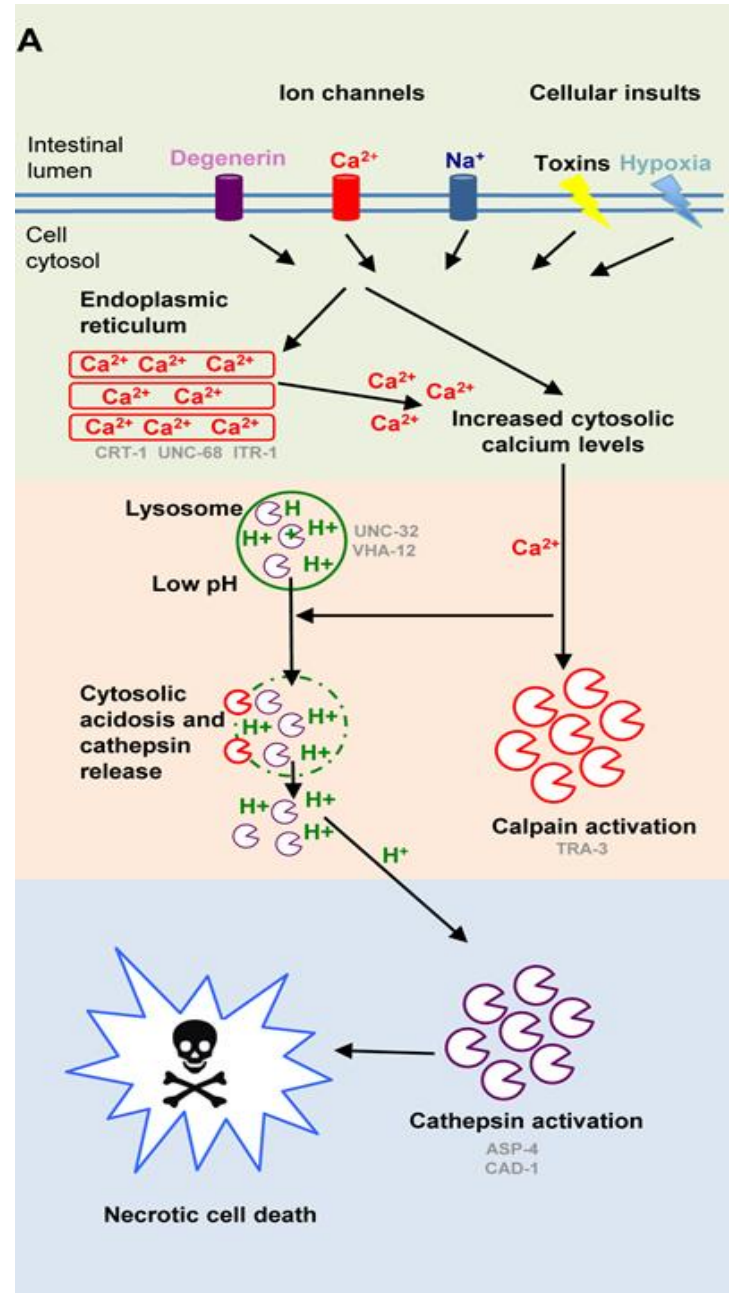

Fig. 1. Necrotic Cell Death Pathway reproduced from Coburn, et al. [1]

It has been shown in C. elegans nematodes that organismal death is accompanied by a burst of blue "death fluorescence" (DF) [1; Fig. 2]. This DF is generated by the necrotic cell death pathway, marking a wave of intestinal cell death [1]. Prior to the burst of DF, aging worms also exhibit increasing levels of red autofluorescence, the biological cause or significance of which is unknown. These worms also show an age-related increase in green fluorescence if they are treated with the fluorescent marker uranin [Fig. 2]. Because uranin localizes to lysosome-related "gut 
granules" within the C. elegans intestine and its fluorescence is dequenched upon lysosomal permeabilization [1], uranin staining provides a mechanism to quantify and study the lysosomal damage component, LMP, of the necrotic cell death pathway.
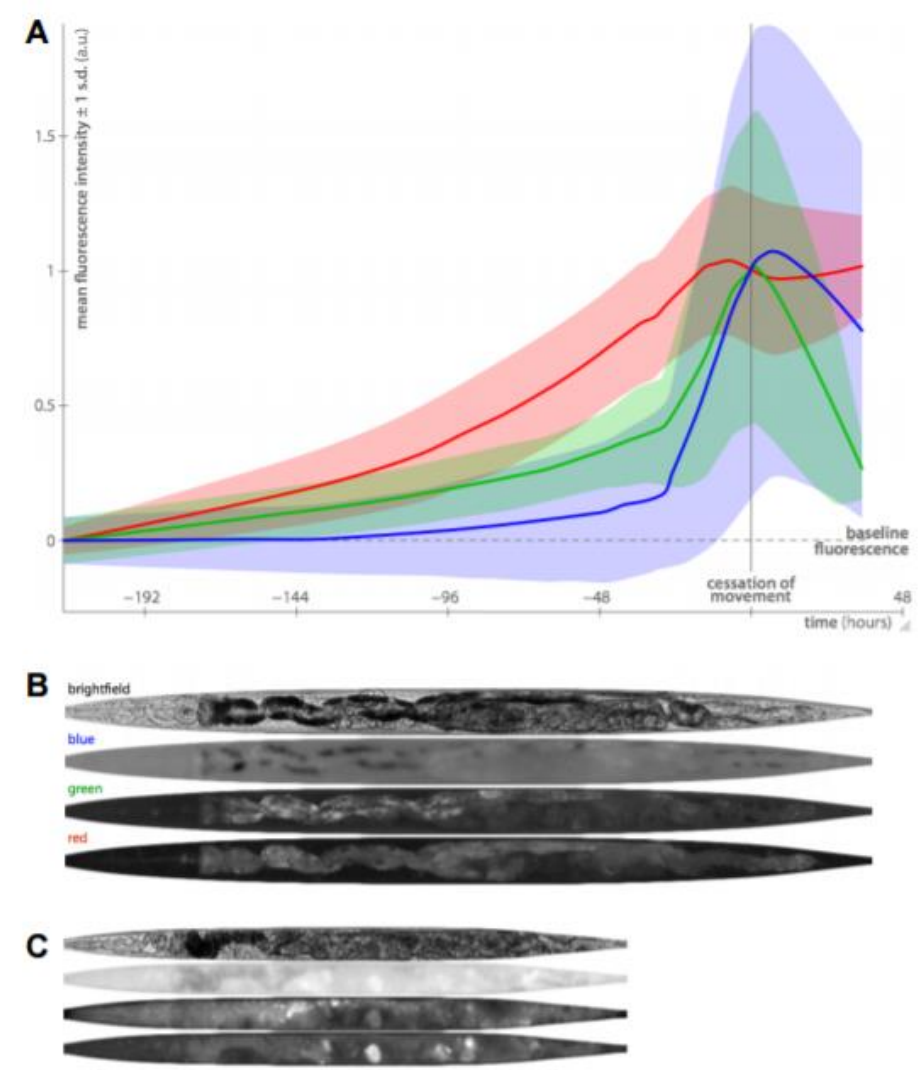

Fig. 2. Other sources of fluorescence increase at death in C. elegans, reproduced from Coburn, et al. (Fig. S5) [1]. (A) Average levels of fluorescence normalized to time of death, viewed under red, blue, and green filter sets [1]. (B and C) Typical images of worms prior to death (B) and during death $(\mathrm{C})$ in each fluorescence band, showing altered spatial distribution of the fluorescence [1]. 
C. elegans mitochondrial (Mit) mutants, which have disrupted mitochondrial electron transport chain (ETC) functionality, have been described by some as "slow-living". That is, despite their reduced ETC efficiency, they are surprisingly long lived, with some Mit mutants living longer than wildtype animals despite comparatively delayed and lower fecundity and higher reactive oxygen species (ROS) levels [9]. It has been hypothesized that Mit mutants' long-lived phenotype arises from differences in their metabolism. Specifically, they compensate for their ETC deficiencies by using alternate ATP pathways that do not require oxygen as a terminal electron acceptor. In support of this hypothesis, experimental findings have shown that several Mit mutants display marked resistance to severe oxygen deprivation and hypoxia [9].

This project aimed to determine whether the "slow living" phenotypes commonly associated with mitochondrial electron transport chain dysfunction (i.e., high endogenous oxidative stress, low reproductive output, and increased longevity) is accompanied by alterations in age-correlated fluorescence patterns or LMP specifically. We used a well-studied C. elegans electron transport chain Mit mutant, gas-1, to examine whether mitochondrial dysfunction has an effect on age-related patterns of subcellular damage and autofluorescence within the context of necrotic cell death.

\section{Methods:}

\section{Nematode Strains:}

This study used the following strains of $C$. elegans nematode: Bristol N2 (standard laboratory wildtype), the ETC mutant gas-1 (fc21), and 15 gas-1 "recovery lines" described below. The gas-1 mutant was obtained from the Caenorhabditis Genetics Center (GCC) at the University 
of Minnesota and then backcrossed to the N2 wildtype strain for several generations to generate an isogenic mutant strain. This was necessary because the chemical mutagen employed to create the gas-1 mutation, ethyl methane sulfonate (EMS), would have causes mutations in other regions. All nematode strains were kept at -80 degrees Celsius prior to thawing.

\section{Experimental Evolution:}

This project took advantage of a previous experiment conducted in the Suzanne Estes lab (Department of Biology) at Portland State University. In that experiment, the aforementioned recovery lines were generated through laboratory adaptation. 24 replicate lines of the gas-1 mutant strain were maintained by serial transfer in large population size $(n=1000)$ for 60 generations. The resulting lines evolved higher fitness and survival compared to their gas-1 progenitor. The recovery lines fell into two categories of high and low fitness relative to one another, with the high fitness class experiencing high fecundity relative to the low fitness class (Fig. 1). Furthermore, it was noted that males, which typically comprise $<1 \%$ of nematode populations, became much more frequent ( $50 \%$; Jennifer Sullins, pers. comm.) during experimental evolution. With few exceptions, recovery lines that grouped into the high fitness class were the same as those that evolved high male frequency and presumably increased rates of outcrossing (Fig. 3). 


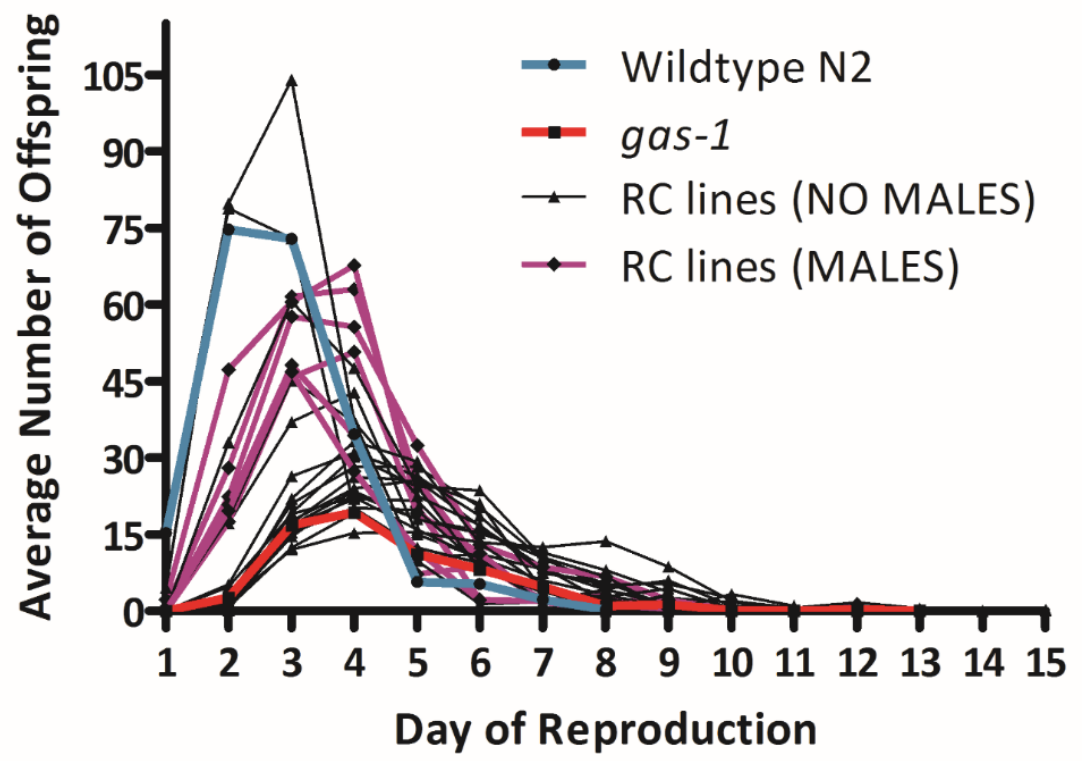

Fig.3. Reproductive schedules for $\mathrm{N} 2$ and gas-1 progenitor strains and gas-1 recovery lines evolved for 60 generations at large population size. Recovery lines fell into high or low fitness classes, a categorization strongly correlated with the presence of males (and presumed frequency of outcrossing). High fitness lines tended to have males present while low fitness lines did not. (Figure provided by Stephen Christy.) [10].

Nematode Sample Preparation:

Prior to all experimentation, each nematode line was thawed and allowed to recover from freezing for two generations. Lines were then age synchronized via bleach treatment. gas- 1 worms were treated 24 hours prior to $\mathrm{N} 2$ and recovery lines to compensate for differences in developmental rate. 
Each strain was divided into a treatment (uranin stained) and control (unstained) group. Target treatment sample sizes were 20 nematodes per line, and control sample sizes aimed to be 5 nematodes per line. For treatment nematodes, 100 microliters of a $20 \mathrm{mg} / \mathrm{ml}$ fluorescein sodium salt (uranin) dye solution was added topically to NGM agar plates seeded with OP50-1 E.coli. Worms of each line were then transferred to the treated plates via a platinum wire pick using sterile technique, and left to feed on the dyed E.coli for 3 hours. The dyed worms were then transferred via pick to undyed seeded NGM agar plates and left for one hour. Control worms remained on undyed NGM seeded plates for the duration of this period. Both treatment and control worms were picked onto microscopy slides with an agar pad and paralyzed using 5-10 microliters of a 1:5 levamisole: M9 solution. Cross-contamination among lines was avoided through ethanol/flame sterilization of the metal wire tool used to transfer worms. To ensure that worms being imaged on day 3 and day 8 were of the original age-matched populations and not subsequent offspring, worms of each line were transferred daily to new seeded NGM plates post day 0 imaging.

\section{Imaging and Image Analysis:}

All lines were imaged 48 hours after bleach age-synchronization of N2 and recovery lines, designated as Day 0. This time point corresponds to roughly the larval stage 4 (L4) period just prior to the young adult molt at which point nematodes are reproductively mature. Imaging took place at three time points: Day 0, 3 days post initial imaging, designated as Day 3, and eight days post initial imaging, designated as Day 8. A Leica confocal microscope was used to capture green (uranin) fluorescence using a GFP laser, red autofluorescence using a TRITC laser, and blue fluorescence (not reported here) using a DAPI laser. Fluorescent and brightfield images were 
captured using a 5X objective for all treatment and control individuals. Using ImageJ software (NIH), the body of each individual was manually outlined on the brightfield images and then transposed onto the corresponding fluorescent images. Bean mean and maximal fluorescence intensity, reported in "relative fluorescent units (RFU)" based on pixel intensity, within that designated area. To control for background fluorescence, an adjusted maximum and adjusted mean fluorescence value were also calculated by subtracting the average and maximum fluorescence values of the strain-specific control (untreated) worms from the treatment line worms.

\section{Statistical Analysis:}

A total of 427 nematodes were imaged across three time points, for a total 1281 images analyzed. All green fluorescence analyses were conducted using log10-transformed raw fluorescence values. Although this transformation failed to achieve normality (Shapiro-Wilke W $=0.9897, \mathrm{p}<0.0001)$, it provided a marked improvement over the highly skewed distribution of the control-adjusted scores. We first tested for differences between the two control progenitor strains using a two-factor ANOVA with model $\mathrm{Y}=$ control treatment (i.e., N2 or gas-1) + day + (treatment $\mathrm{x}$ day), and then among experimental lines and across days of life using the model: $\mathrm{Y}=$ $\mu+$ line + day $+($ line $x$ day $)+\varepsilon$, where line is random effect and day was a fixed effect. We assessed pairwise differences using post-hoc Dunnet's comparisons with N2 wildtype as the control. We also tested whether variance in RC line fluorescence patterns with age could be explained by fitness classification using two-factor ANOVAs testing the model: $\mathrm{Y}=\mu+$ day + fitness classification $+($ day $\mathrm{x}$ fitness classification $)+\varepsilon$. The same analyses were performed for red fluorescence except that control-adjusted mean values were used, following the same reasoning: 
analyses using these data revealed no effect of assay block and were more normally distributed than all other metrics.

Lastly, we investigated line mean correlations between age-specific red and green fluorescence and between relative fitness and fluorescence using linear regression. All statistical analysis was performed using JMP (SAS) v. 13.

\section{Results:}

First, the gas-1 mutant progenitor of the recovery lines exhibited divergence from N2 in terms of overall amount and age-related patterns of green fluorescence (Figs. 4-5), but less so for red fluorescence (Figs. 6-7). For uranin (green) fluorescence, significant effects of control strain, day and the interaction of the two were revealed (Table 1). Specifically, the gas-1 mutant was overall more fluorescent than $\mathrm{N} 2$, but each control strain exhibited a different age-related pattern such that N2 fluorescence increased between Days 0 and 3 and then plateaued, and gas- 1 showed reduced fluorescence during Day 3 with recovery of Day 0 levels by Day 8 (Figs. 4-5).

\begin{tabular}{lllll}
\multicolumn{4}{l}{$\begin{array}{l}\text { Table 1. ANOVA results for N2 vs. gas- } 1 \\
\text { Source }\end{array}$} & uranin fluorescence. \\
& $\begin{array}{l}\text { Sum of } \\
\text { Squares }\end{array}$ & F Ratio & p-value \\
\hline Treatment & 1 & 8.985893 & 56.0295 & $<.0001^{*}$ \\
Day & 2 & 2.449931 & 7.6380 & $0.0006^{*}$ \\
Treatment*Day & 2 & 10.436955 & 32.5386 & $<.0001^{*}$
\end{tabular}

Conversely, both control strains exhibited age-related increases in red autofluorescence (Figs. 6-7), particularly on Day 8 of measurement. This increase was slightly more pronounced in gas-1 as indicated by the marginally significant treatment $\mathrm{x}$ day interaction term (Table 2). 
Although control treatment did not account for a significant fraction of the variation in this model, a post-hoc test revealed that Day 3 gas-1 fluorescence was significantly greater than that of N2.

Table 2. ANOVA results for N2 vs. gas-1 red autofluorescence.

$\begin{array}{lrrrr}\text { Source } & \text { DF } & \begin{array}{r}\text { Sum of } \\ \text { Squares }\end{array} & \text { F Ratio } & \text { p-value } \\ \text { Treatment } & 1 & 0.0141391 & 0.3551 & 0.5516 \\ \text { Day } & 2 & 2.7233941 & 34.1971 & <.0001^{*} \\ \text { Treatment*Day } & 2 & 0.2287383 & 2.8722 & 0.0578\end{array}$

Recovery (RC) lines assayed exhibited divergence from the gas-1 ancestor, with a weak tendency toward lower fluorescence across days compared to the ancestor, but no strong directionality to their pattern of mean divergence was observed (Fig. 4). Analyses revealed significant effects of line, day of measurement and the interaction between the two ( $\mathrm{F}$ tests, $\mathrm{p}<$ 0.001). RC lines also diverged from each other in mean age-related fluorescence. Specifically, analysis revealed significant among-line variance for fluorescence at each time point $(\mathrm{F}$ tests; $\mathrm{p}<$ 0.0001) that tended to increase with age in both assays; the same pattern was observed for withinline (environmental) variance (not shown). 


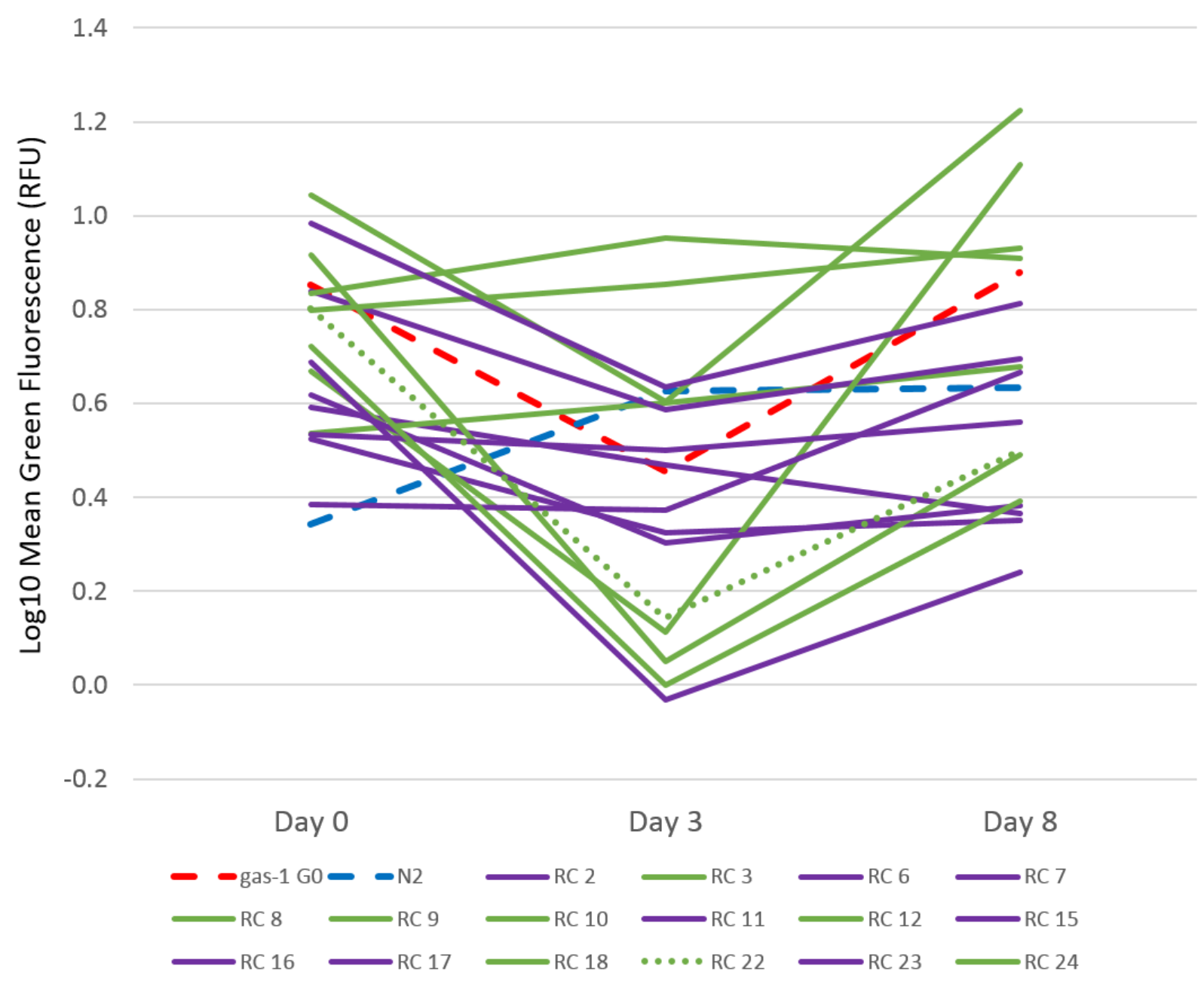

Fig.4. Average green (uranin) fluorescence of replicate N2 (blue) and gas-1 (red) progenitor lines alongside gas-1 recovery lines across three imaging time points with high fitness recovery lines in green and low fitness recovery lines in purple. Highest-fitness recovery line RC22 is highlighted for comparison.

$\mathrm{RC}$ line mean green fluorescence was related to the relative fitness they achieved after 60 generations of laboratory adaptation (Table 3). Both high- and low-fitness groups shared the dip in Day 3 fluorescence exhibited by the gas-1 ancestor (Fig. 5), but fluorescence of high-fitness RC 
lines shows a tendency to rebound to higher ancestral levels on Day 8 while low-fitness RC lines remained lower (and similar to N2) on Day 8. High-fitness RC lines were not distinguishable on average from low-fitness lines on Day 3, but were significantly more fluorescent on Days 0 and 8 (Tukey's HSD, $\alpha=0.05$ ). There is, however, considerable variation (Fig. 4); for instance, the RC line that achieved highest fitness during the experiment, RC22 (highlighted in Fig. 4), follows the pattern of low-fitness RC lines.

Table 3. ANOVA results of age-related uranin fluorescence in high-vs. low-fitness gas-1 RC lines.

$\begin{array}{lrrrrr}\text { Source } & \text { Nparm } & \text { DF } & \begin{array}{r}\text { Sum of } \\ \text { Squares }\end{array} & \text { F Ratio } & \text { Prob }>\text { F } \\ \text { Treatment } & 1 & 1 & 2.064661 & 17.4448 & <.0001^{*} \\ \text { Day } & 2 & 2 & 12.403059 & 52.3981 & <.0001^{*} \\ \text { Treatment*Day } & 2 & 2 & 2.237889 & 9.4542 & <.0001^{*}\end{array}$




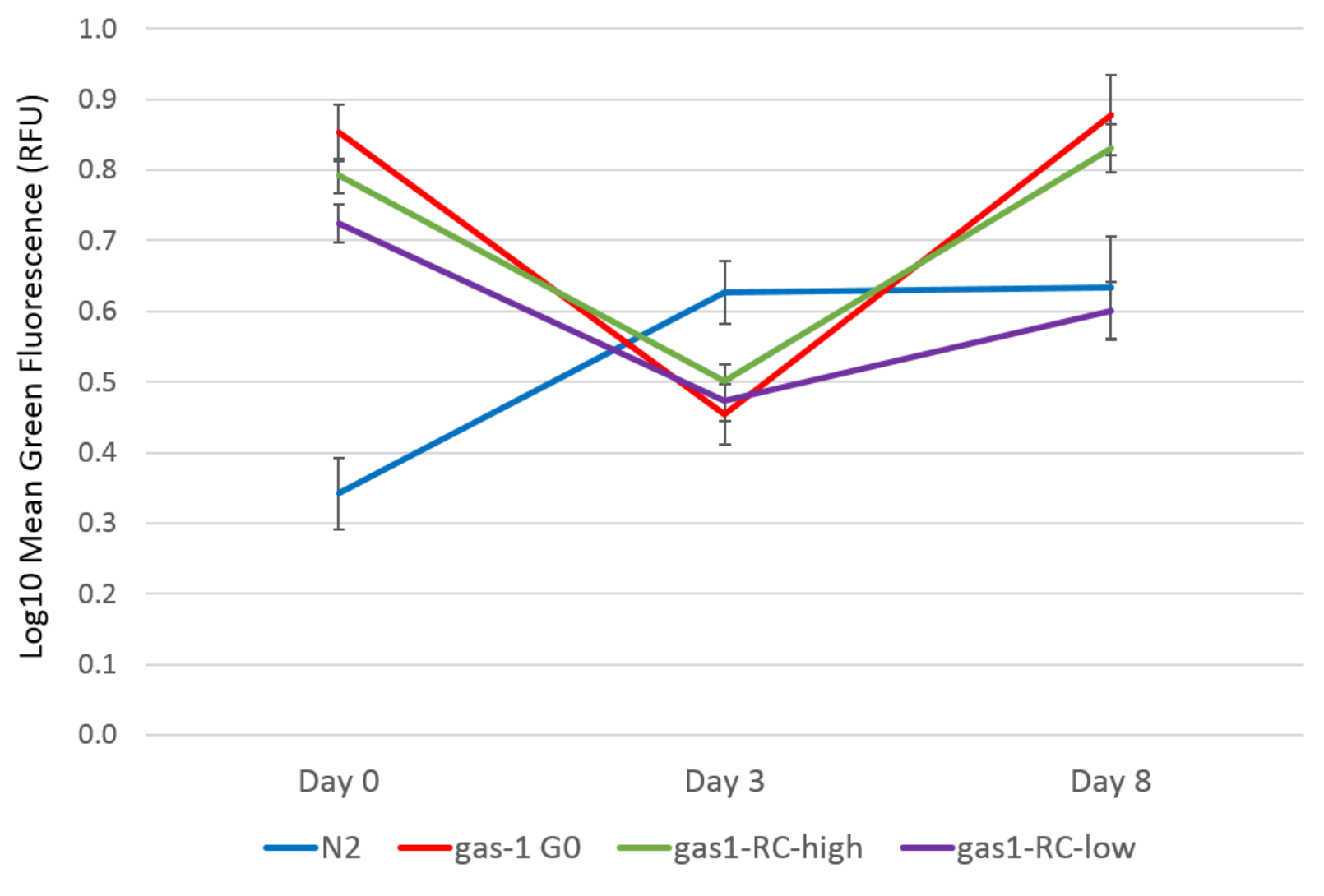

Fig. 5. Average green (uranin) fluorescence of $\mathrm{N} 2$ and gas-1 progenitor lines alongside high-fitness (green) and low-fitness (purple) gas-1 recovery line groups. Error bars are 1 S.E.M.

For red fluorescence, RC lines mainly cluster around the two ancestral strains, but with considerable variation in pattern. Analyses revealed significant effects of line, day of measurement and the interaction between the two (F tests, $\mathrm{p}<0.001$ ). As show in Fig. 4, many RC lines show a pattern of increased fluorescence between Days 0 and 3, unlike the patterns of the control strains. The highest-fitness line, RC22, exemplifies this pattern (highlighted line in Fig. 6). 


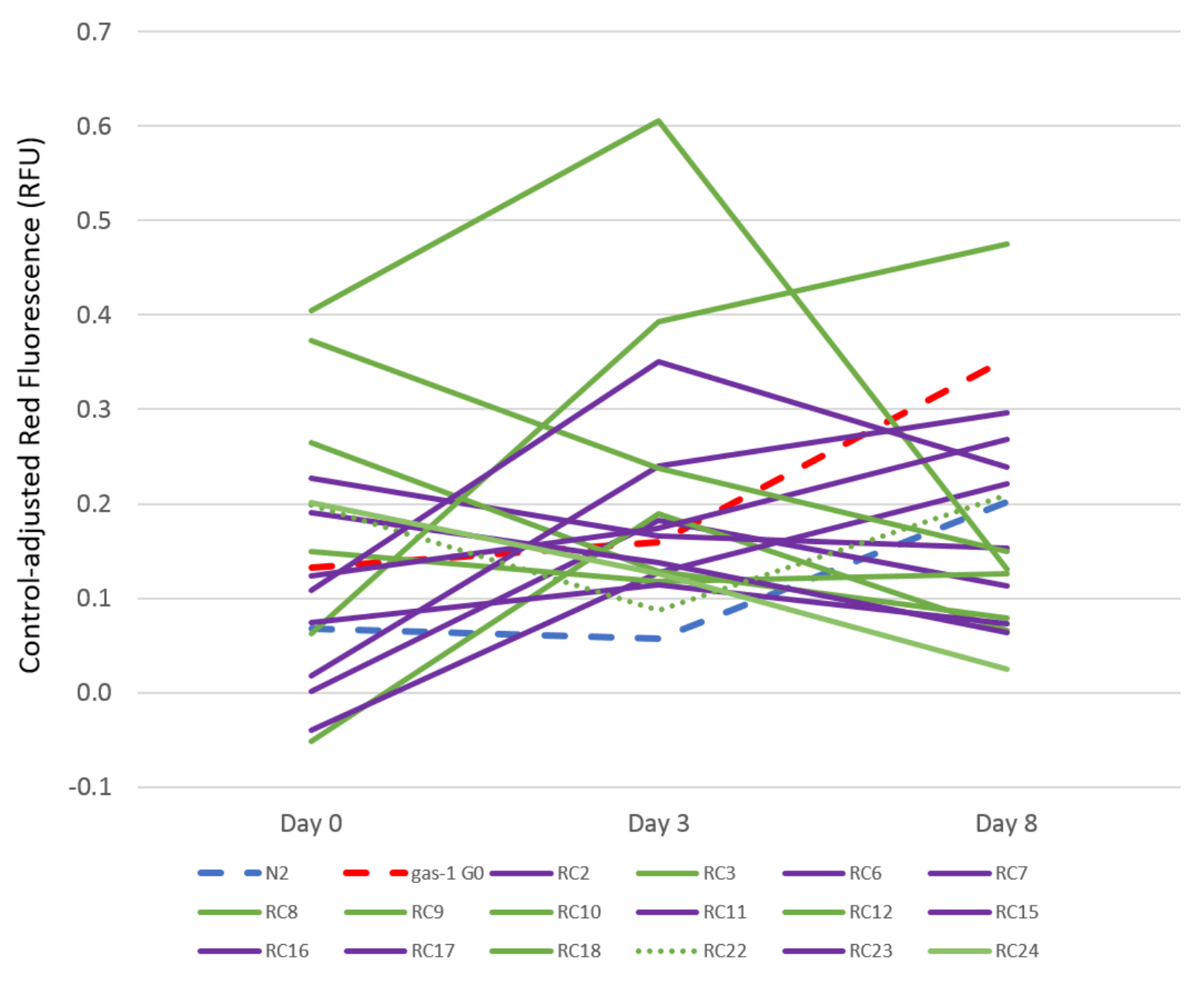

Fig. 6. Average red fluorescence of replicate N2 (blue) and gas-1 (red) progenitor lines alongside gas-1 recovery lines across three imaging time points with high fitness recovery lines in green and low fitness recovery lines in purple. Highest-fitness recovery line RC22 is highlighted for comparison.

Fitness class was also related to RC line autofluorescence (Table 4; Fig. 7). Both groups show a general pattern of increase to Day 3, but while the low-fitness class plateaus at Day 8, the high fitness class declines to approximately its Day 0 levels (Fig. 5; Tukey's HSD, $\alpha=0.05$ ). 
Table 4. ANOVA results of age-related red autofluorescence in high- vs. low-fitness gas-1 RC lines.

$\begin{array}{lrrrrr}\text { Source } & \text { Nparm } & \text { DF } & \begin{array}{r}\text { Sum of } \\ \text { Squares }\end{array} & \text { F Ratio } & \text { Prob }>\text { F } \\ \text { Treatment } & 1 & 1 & 0.5877171 & 11.9483 & 0.0006^{*} \\ \text { Day } & 2 & 2 & 1.7295004 & 17.5803 & <.0001^{*} \\ \text { Treatment*Day } & 2 & 2 & 0.5396544 & 5.4856 & 0.0043^{*}\end{array}$

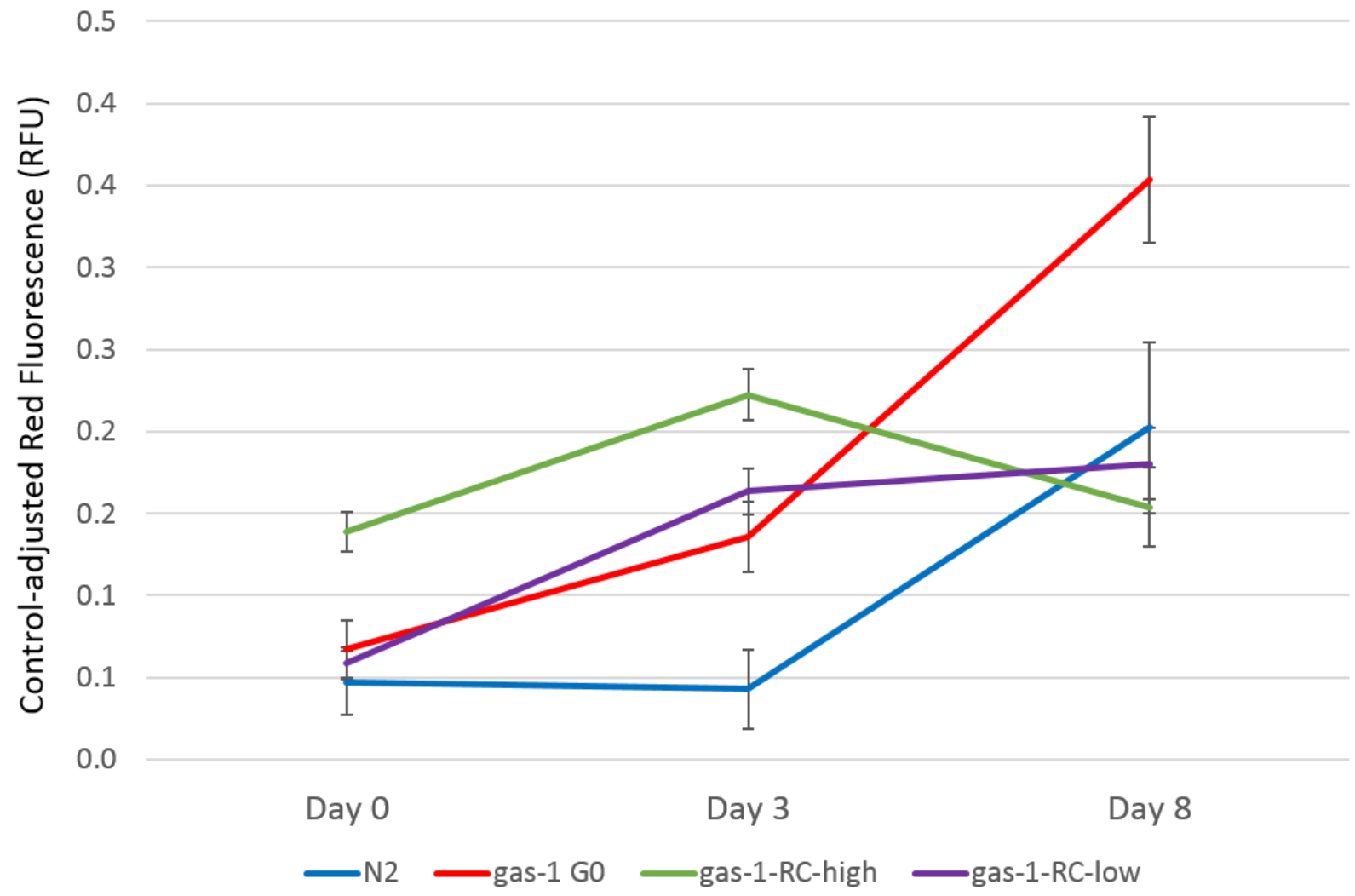

Fig. 7. Average red fluorescence of N2 and gas-1 progenitor lines alongside high-fitness (green) and low-fitness (purple) gas-1 recovery line groups. Error bars are 1 S.E.M.

Finally, we calculated RC-line mean correlations between relative fitness (using data from Christy et al. 2017) and mean green and mean red fluorescence on each day of measurement, and 
found only one statistically significant relationship. The correlation between Day 8 green fluorescence and relative fitness was only weakly positive $(r=0.28)$ and nonsignificant. However, visual inspection of the bivariate relationship between these traits (Fig. 8) indicated the presence of an outlier line (confirmed by formal analysis), RC22, the line that achieved the highest level of evolved fitness-exceeding that of wildtype N2. RC22 fluorescence was low for its level of relative fitness compared to other lines. Removal of RC22 from the dataset resulted in a stronger and significantly positive correlation between Day 8 fluorescence and relative fitness $(r=0.614$, $\mathrm{p}=0.028)$.

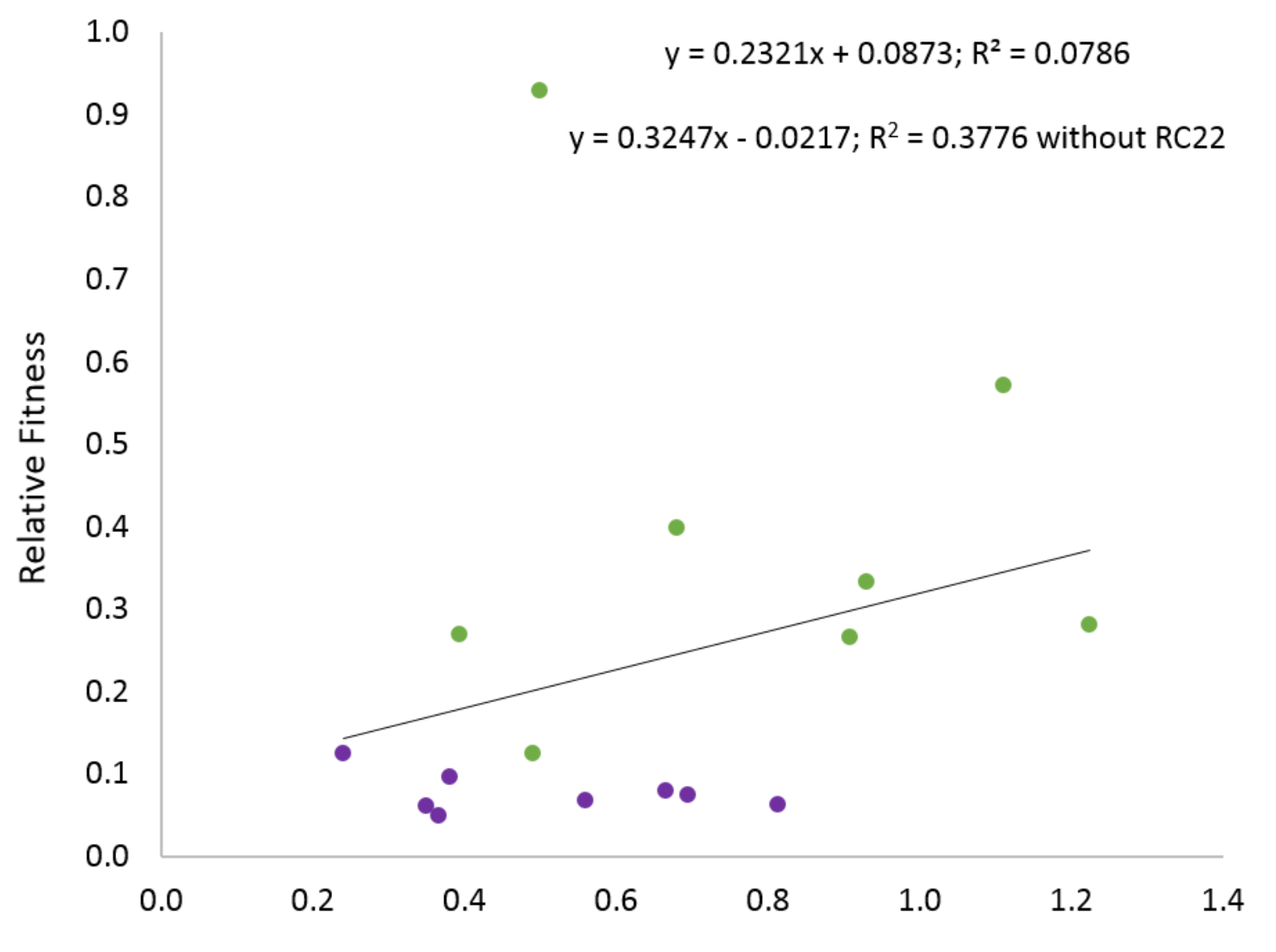

Day 8 Log10 Mean Green Fluorescence (RFU) 
Fig. 8. Bivariate plot of recovery line fitness relative to $\mathrm{N} 2$ wildtype and average uranin (green) fluorescence on Day 8 of measurement. Green points are means for high-fitness lines; purple points are means for low-fitness line. The outlying green point at top represents RC22. The equation and $\mathrm{R}^{2}$ value for the regression line is shown at top; the second equation and $\mathrm{R}^{2}$ value were calculated with RC22 removed.

\section{Discussion:}

Although not reported here, blue DF, which was measured on all of the same individuals, remained undetectable across worm age in both control strains; therefore, we did not further analyze DF for the evolved lines. If our experiment had continued until nematode death like that of Coburn, et al. [1], we expect that the previously observed DF spike would have occurred at approximately the same time for both strains.

Conversely, while green (uranin) fluorescence increased in older wildtype nematodes as previously observed, gas-1 animals tended to exhibit higher levels of uranin fluorescence across days of measurement - with a marked dip for middle-aged (Day 3) animals. Thus, the gas-1 mutation may cause animals to experience mostly greater levels of lysosomal membrane permeability associated with necrotic death compared to wildtype. The underlying nature of the

dip in Day 3 fluorescence is unknown, but may be related to changes in mitochondrial abundance and activity across the gas-1 nematode life cycle that differ from N2.

Age-related uranin fluorescence for the evolved recovery lines show a vaguely similar pattern to the gas- 1 ancestor in that they reproduced gas-1's dip in Day 3 fluorescence; however, there was much variation in mean fluorescence across days among the independently evolved 
recovery lines. The only other pattern of note was that recovery lines showed increased variance in fluorescence levels with age, with lines from the higher fitness class trending slightly toward higher levels of green fluorescence compared to lines from the lower fitness class. This pattern was borne out in our investigation of line-mean correlations. Although neither fluorescence type was strongly related to the relative fitness achieved by a line after 60 generations of laboratory adaptation/recovery, the one exception was for Day 8 uranin fluoresence. For these animals, higher fitness lines showed a weak, but significant tendency toward higher late-life fluorescence-but only if the outlier line, RC22, was omitted. This line achieved the highest recovery fitness, outcompeting even N2 wildtype. It exhibited much lower fluorescence than predicted based on the regression line. If this trend is biologically relevant, it may be due to the earlier reproduction seen in the higher fitness class lines compared to the lower fitness class lines-if early reproduction equates with "faster living" or more rapid biological aging. While more work would be required to understand this pattern, there may be a tradeoff between reproduction and an underlying mechanism such as necrotic cell death. It may be that RC22 evolved a means to break the correlation between higher fluorescence and earlier reproduction seen in the higher fitness lines.

Red autofluoresence was previously observed to show a more gradual and linear increase with age in post-reproductive wildtype C. elegans than either blue DF or uranin fluorescence [1]. We found the same pattern for both $\mathrm{N} 2$ and gas- 1 mutant strains, with gas-1 being uniformly more fluorescent than wildtype (not significant). If it is true that red fluorescence is a better indicator of chronological age, it may be that gas-1, which is slightly longer-lived than $\mathrm{N} 2$, nonetheless exhibits more rapid senescence or "unhealthy aging". Similar to uranin fluorescence, the gas-1 recovery lines exhibited considerable among-line variance, but revealed a rather different pattern of change with age than either their gas-1 ancestor or N2 wildtype. Recovery lines from both high and low 
fitness classes exhibited similar patterns of age-correlated change in red autofluorescencenamely, an early-life increase followed by a plateau. It is thus possible that the recovery lines are aging differently either control strain. It is noteworthy that, with only one exception, all recovery lines exhibited lower Day 8 red fluorescence than gas-1.

Lastly, our investigation of recovery line-specific correlations between the two forms of fluorescence (uranin and red) revealed no discernable patterns. The uranin fluorescence of a particular line was not related on average to its red fluorescence, suggesting that the two fluorescence types reflect different biological phenomena.

We conclude that oxidative stress caused by gas- 1 mitochondrial dysfunction is associated with increased age-related subcellular damage and autofluoresence, even though this fluorescence has little bearing on total longevity; i.e., gas-1 exhibits unhealthy aging. Second, although the adaptive evolution experienced by the gas-1 recovery lines was accompanied by highly variable changes in both types of fluorescence, there is some indication that the oldest worms surveyed here (i.e., Day 8 time point) more closely resembled wildtype levels of fluorescence. Thus, adaptation may have been accompanied (or driven) by recovery of a more wildtype pattern of biological aging. 


\section{References:}

1. Coburn, C., Allman, E., Mahtani, P., Benedetto, A., Cabreiro, F., Pincus, Z., .. . Gems, D. (2013). Anthranilate Fluorescence Marks a Calcium-Propagated Necrotic Wave That Promotes Organismal Death in C. elegans. PLoS Biology PLoS Biol, 11(7)

2. Kerr JF, Wyllie AH, Currie AR (1972) Apoptosis: a basic biological phenomenon with wide-ranging implications in tissue kinetics. Br J Cancer 26: 239-257.

3. Golstein P, Kroemer G (2007) Cell death by necrosis: towards a molecular definition. Trends Biochem Sci 32: $37-43$.

4. Luke CJ, Silverman GA (2011) Necrotic cell death: harnessing the Dark side of the Force in mammary gland involution. Nat Cell Biol 13: 197-199.

5. McCall K (2010) Genetic control of necrosis - another type of programmed cell death. Curr Op Cell Biol 22: 882-888.

6. Zong WX, Thompson CB (2006) Necrotic death as a cell fate. Genes Dev 20: 1-15.

7. Kroemer G, Galluzzi L, Vandenabeele P, Abrams J, Alnemri ES, et al. (2009). Classification of cell death: recommendations of the Nomenclature Committee on Cell Death 2009. Cell Death Differ 16: 3-11.

8. Mattson MP (2007) Calcium and neurodegeneration. Aging Cell 6: 337-350.

9. Butler JA, Ventura N, Johnson TE, Rea SL. Long-lived mitochondrial (Mit) mutants of Caenorhabditis elegans utilize a novel metabolism. FASEB J. 2010;24:4977-4988. 
10. Christy S. (2017) Adaptive evolution under favorable and unfavorable population genetic conditions in Caenorhabditis elegans. Master's Thesis; Portland State University. 